\title{
Numerische Begriffe
}

\section{3-D Secure}

3-D Secure ist ein XML-basiertes $\rightarrow$ Protokoll, das als zusätzliche Sicherheitsprüfung für Online-Zahlungskartentransaktionen dient. Das Kreditkartenunternehmen Visa hat das Verfahren erstmals eingesetzt, um die Sicherheit der $\rightarrow$ Authentisierung von Kartenzahlungen über das Internet zu verbessern und bietet es als Dienstleistung unter der Bezeichnung „Verified by Visa“ an.

\section{2}

Die Zahl 42 ist im $\rightarrow$ Fintech- und $\rightarrow$ Start-upUmfeld eine häufig anzutreffende Antwort auf die Frage nach dem Sinn des Lebens oder ,weshalb man etwas macht, wie man es macht“. Sie geht zurück auf den Science-Fiction-Roman von Douglas Adams „Life, the universe and everything“" aus dem Jahr 1982.

\section{G}

Mobilfunktechnologie der fünften Generation, welche die Datenübertragungsrate der Vorgänger-Technologie 4G (LTE). von bis zu $100 \mathrm{MBit} / \mathrm{s}$ auf bis zu $10 \mathrm{GBit} / \mathrm{s}$ erhöhen soll. Diese Ge- schwindigkeitsverbesserungen bilden die Grundlage von neuen Anwendungsfeldern wie dem $\rightarrow$ Pervasive Computing und dem Internet der Dinge $(\rightarrow \mathrm{IoT})$. Wie bei Infrastrukturgütern bekannt, existiert bei der Verbreitung der neuen Netzwerktechnologie ein Zusammenhang zwischen der möglichst deckenden Bereitstellung der Netzwerkinfrastruktur und der Verfügbarkeit 5G-fähiger Endgeräte einerseits sowie der 5G-fähigen Anwendungen bzw. $\rightarrow$ Services andererseits. Während die technologische Infrastruktur zunehmend gegeben ist, besteht noch ein Engpass bei den Anwendungen, die z. B. im Finanzbereich ortsbezogener $\rightarrow$ Services $(\rightarrow$ LBS) sowie $\rightarrow$ Smart Services, die Nutzung aller Interaktionskanäle ( $\rightarrow$ Omni-Channel) und die $\rightarrow$ Echtzeitverarbeitung im $\rightarrow$ Mobile Banking umfassen könnten (z. B. bezüglich Sicherheit und regulatorischer Vorgaben wie im Bereich $\rightarrow$ AML). Parallel zum Ausbau der 5G-Infrastruktur erfolgt bereits die Forschung zum nachfolgenden 6G-Standard, der Datenraten bis zu einem TBit/s und damit die Vernetzung von Alltagsgegenständen sowie zahlreiche weitere Anwendungsmöglichkeiten auch für die Finanzwirtschaft bieten soll. 